\title{
PLR-TV: patch-based low rank with spatio- temporal total variation constraints for ungated myocardial perfusion CMR
}

\author{
Ganesh Adluru*, Edward V DiBella \\ From 17th Annual SCMR Scientific Sessions \\ New Orleans, LA, USA. 16-19 January 2014
}

\section{Background}

Ungated myocardial perfusion is a promising alternative for simplifying CMR protocols [1]. Spatio-temporal total variation (TV) constrained reconstruction with radial undersampling was used in a pilot study [2]. And TV constraints combined with a low-rank constraint have shown improvement in some cases for gated perfusion imaging [3]. However, the temporal total-variation constraint may not effectively resolve undersampling artifacts in ungated studies with significant cardiac and respiratory inter-frame motion. A patch-based low-rank method can more effectively exploit redundancies in a dynamic dataset in the form of spatio-temporal patches rather than one large rectangular matrix with an entire image per column. The patch-based method (also termed locally low rank or blockwise low rank) was recently shown to improve upon the standard low-rank constraint in cardiac cine and perfusion imaging $[4,5]$. Here we adapt a patch-based low-rank method for ungated myocardial perfusion imaging and use it in conjunction with TV constraints.

\section{Methods}

Ungated perfusion data was acquired for eight patients on a Siemens $3 \mathrm{~T}$ Verio scanner using a saturation recovery sequence [2] at rest and at pharmacologically induced stress with TR $=2.2 \mathrm{msec}, \mathrm{TE}=1.2 \mathrm{msec}$, FOV = $280 \mathrm{~mm} 2$, matrix size $=144 \times 20$. Five slices were acquired in $250 \mathrm{msec}$. Patch-based low-rank with spatiotemporal TV constraints was applied in a Projection Onto Convex Sets (POCS) based alternating framework [6,7] while enforcing data fidelity. We used overlapping patches that are approximate circles on Cartesian grid as shown in
Figure 1 with a radius of 8 pixels. The overall amount of spatial overlap in the image was $<60 \%$.

\section{Results}

Figure 2 compares the results of the PLR-TV with TV reconstructions. PLR-TV images have less pixelation artifacts than the TV reconstructions and have improved delineation of papillary muscles and myocardial boundaries especially in post-contrast frames.

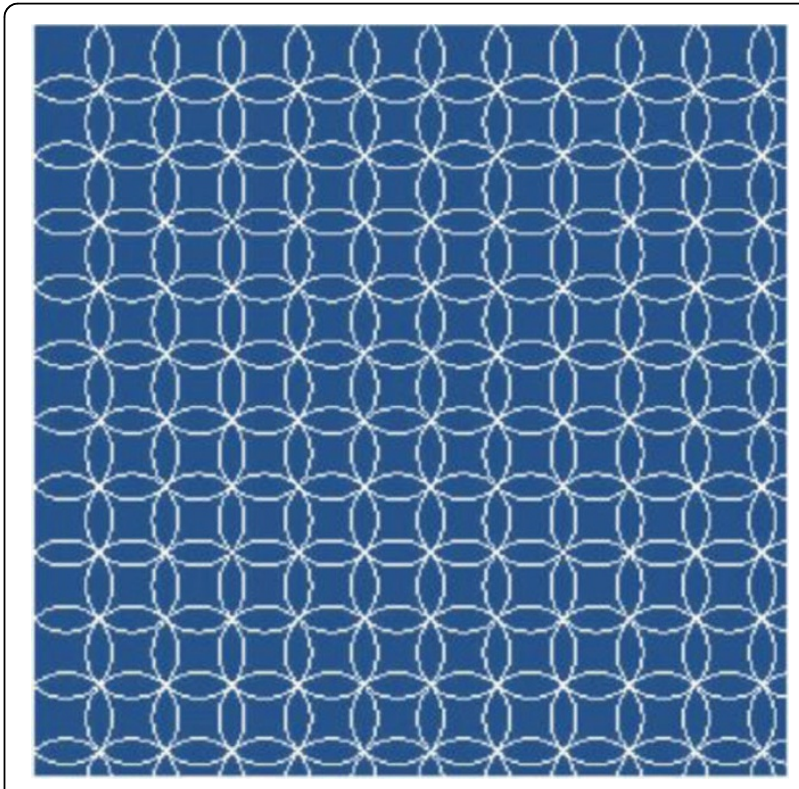

Figure 1 Image showing approximately circular patches that are overlapping on a Cartesian grid for PLR constraint.

Radiology, University of Utah, Salt lake city, Utah, USA 


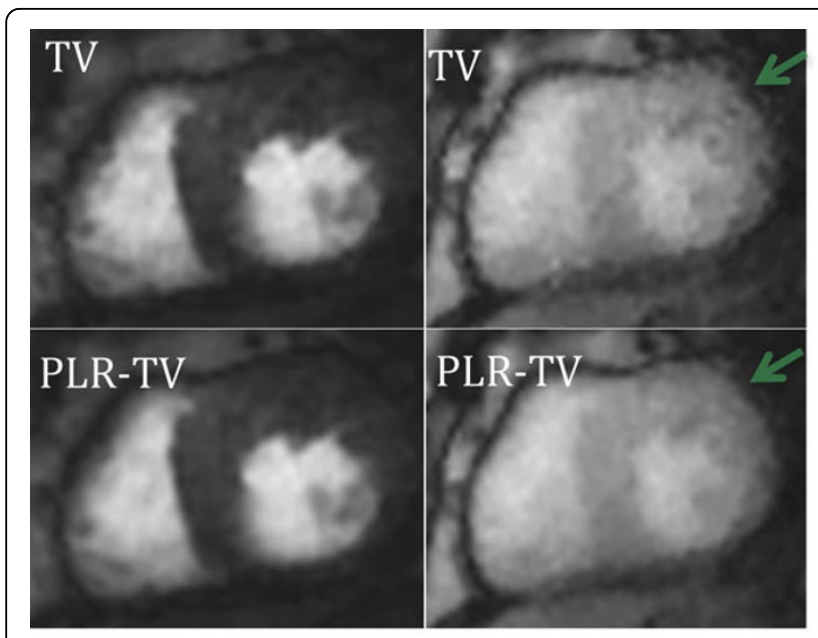

Patient \#1

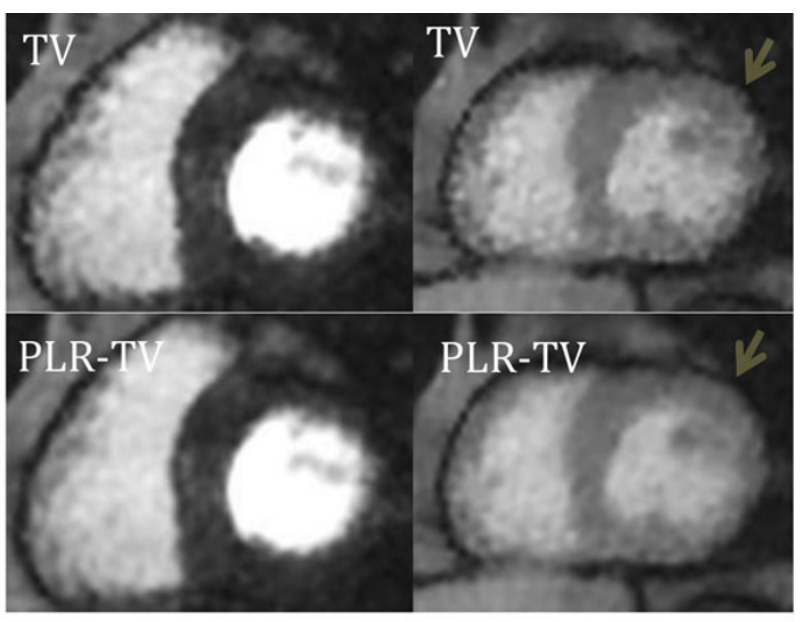

Patient \#2

Figure 2 Comparison of reconstructed image quality for ungated radial perfusion imaging for two patient datasets. Near systolic frames pre and post contrast are shown. Images labeled TV are reconstructions with spatio-temporal TV constraints and those labeled with PLR-TV are corresponding images with patch-based low ran and spatio-temporal TV constraints.

\section{Conclusions}

PLR-TV is a promising approach for reconstructing undersampled radial ungated data. While the PLR constraint exploits the low rank property effectively in the ungated images, TV constraints can help preserve any contrast loss from the PLR, making the hybrid method more effective than either constraint alone in the presence of motion. Here we chose overlapping circular patches over time (so that the Casorati matrix is almost square), although arbitrary shaped patches can be chosen depending on underlying geometrical structures. While adding PLR constraints increases the reconstruction time, we limited the amount of overlapping of the circular patches so as to cover all of the pixels in the image for the PLR constraint. Randomized patch updates and the use of TV reconstructions as initial estimates for the final PLR-TV reconstructions can reduce the computational cost.

\section{Funding}

\section{RO1 HL113224.}

Published: 16 January 2014

$$
\begin{aligned}
& \text { References } \\
& \text { 1. MRM 2012, 67:609-613. } \\
& \text { 2. JCMR 2013, 15:26. } \\
& \text { 3. PMB, to appear 2013. } \\
& \text { 4. ISMRM 2011, \#4371. } \\
& \text { 5. ISMRM 2013, \#4555. } \\
& \text { 6. MRI 2012, 30:1483-1494. } \\
& \text { 7. JMRI 2010, 32:1217-1227. }
\end{aligned}
$$

doi:10.1186/1532-429X-16-S1-W22

Cite this article as: Adluru and DiBella: PLR-TV: patch-based low rank with spatio-temporal total variation constraints for ungated myocardial perfusion CMR. Journal of Cardiovascular Magnetic Resonance 2014 16(Suppl 1):W22.

\section{Submit your next manuscript to BioMed Central} and take full advantage of:

- Convenient online submission

- Thorough peer review

- No space constraints or color figure charges

- Immediate publication on acceptance

- Inclusion in PubMed, CAS, Scopus and Google Scholar

- Research which is freely available for redistribution
Biomed Central 